\title{
Direct numerical simulation of supercritical annular electroconvection
}

\author{
Peichun Tsai, ${ }^{1}$ Zahir A. Daya, ${ }^{2}$ Vatche B. Deyirmenjian, ${ }^{1}$ and Stephen W. Morris ${ }^{1}$ \\ ${ }^{1}$ Department of Physics, University of Toronto, 60 St. George Street, Toronto, Ontario, Canada M5S 1A7 \\ ${ }^{2}$ Defence Research \& Development Canada, 9 Grove Street, Dartmouth, Nova Scotia, Canada B2Y $3 Z 7$
}

(Received 9 March 2007; published 10 August 2007)

\begin{abstract}
We use direct numerical simulation to study electrically driven convection in an annular thin film. The simulation models a laboratory experiment that consists of a weakly conducting, submicron thick liquid crystal film suspended between two concentric electrodes. The film is driven to convect by imposing a sufficiently large voltage across it. The flow is driven by a surface charge density inversion which is unstable to the imposed electrical force. This mechanism is closely analogous to the mass density inversion which is unstable to the buoyancy force in conventional, thermally driven Rayleigh-Bénard convection. The simulation uses a pseudospectral method with Chebyshev polynomials in the radial direction and Fourier modes in the azimuthal direction. The numerical results, which are in good agreement with previous experimental data and theoretical predictions, reveal several insights. The mode competition near a codimension-two point exhibits hysteresis. The primary bifurcation is supercritical for a broad range of fluid and geometrical parameters.
\end{abstract}

DOI: 10.1103/PhysRevE.76.026305

PACS number(s): 47.20.Ky, 47.27.ek

\section{INTRODUCTION}

Convection has long been a playground for investigators of nonlinear dynamics and pattern formation [1]. Highly controlled experiments, accurate simulations, and analytic theoretical analyses can be combined to give an unusually complete picture of the dynamics, particularly in the weakly nonlinear regime [2]. The accumulation of complexity as the driving forces are increased also presents an important route to chaotic and, eventually, fully turbulent flows [3]. Here, we present a direct numerical study of annular electroconvection in a thin film, a model problem that has previously attracted detailed experimental [4-10] and theoretical [11-13] attention. This study is a step toward the development of a numerical code capable of reaching the fully turbulent regime that was recently accessed experimentally $[9,10]$.

Classic convection experiments include Rayleigh-Bénard convection (RBC), the buoyancy-driven instability of a fluid layer heated from below [1], and electrohydrodynamic convection (EHC) in nematic liquid crystals. In the latter, an applied electric field drives a charge density that develops in certain nematic fluids [14].

In the present study, we exploit the unusual properties of smectic liquid crystals which form extremely robust, submicron thick, and freely suspended films. When a constant voltage is applied between the inner and outer edges of an annular film, it convects due to an unstable surface charge distribution that develops near the free surfaces. The film geometry and the experimental setup are shown schematically in Fig. 1. Unlike EHC, this electrical driving mechanism does not rely on the dielectric anisotropy of the liquid crystal. The experiment uses smectic- $A$ phase materials which are isotropic for flows in the plane of the film.

Electroconvection in smectic films shares some of the advantages that $\mathrm{EHC}$ has over conventional RBC: Fast time scales, independence from gravity, and all-electrical transport measurements. However, flow visualization in the thin film is difficult [4]. An important motivation of the present direct numerical study is to enable the visualization of the basic fields.
The linear theory of this instability is well established [11], including for the case of an annular geometry with a superposed shear [13]. The basic mechanism of the instability turns out to be highly analogous to that of RBC, albeit with radial driving forces [15]. The annular geometry gives rise to numerous codimension-two (CoD2) points and secondary bifurcations within the weakly nonlinear regime $[8,13,15]$. Previous experimental work in this regime has shown that the amplitude of convection just above onset is well modeled by a Landau amplitude equation with a cubic nonlinearity [6]. Previous theoretical analyses, using amplitude equations derived from first principles, showed good agreement between experiment and theory [8]. More recent experiments [9] have pushed into the highly nonlinear, turbulent regime where scaling behavior is observed [10]. Direct numerical simulations offer complementary insights into this system.

In this work, we develop a direct numerical simulation using a pseudospectral method with realistic governing equations. This code allows us to extend the range of parameters beyond what is achievable experimentally and into the strongly nonlinear regime which is difficult to treat with analytic theory. In addition, the simulation allows us to visualize all the basic fields and gain new insights into the complex flow dynamics in chaotic and turbulent states that occur at higher electrical forcing.

In this paper, we focus on a detailed numerical study of states near the primary bifurcation and at a CoD2 point, covering a large parameter space in the weakly nonlinear regime. We compare numerical data with experimental and (a)

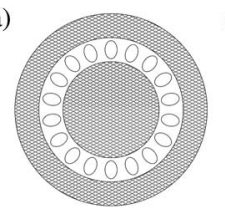

(b)

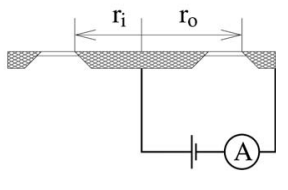

FIG. 1. Schematics of the annular electroconvection experiment: (a) Top view and (b) side view. 
theoretical results. The paper is organized as follows. First, in Sec. II A, we briefly introduce the two-dimensional (2D) annular electroconvection experiment and its phenomenology. Then, in Sec. II B, we describe the physics underlying the mathematical model. In Sec. III we present our numerical method and setup for direct numerical simulations. In Sec. IV, we show some numerical data and compare with previous theoretical and experimental results. We highlight results for small aspect ratios, small dimensionless number $\mathcal{P}$, which is similar to the Prandtl number in RBC, and on the bifurcation dynamics close to a CoD2 point. Section V presents a brief conclusion and outlines the implications of the numerical results. We also discuss the prospect for future applications of the numerical code.

\section{2D ANNULAR ELECTROCONVECTION}

In this section, we introduce the experimental system and briefly describe the basic equations we used to model it. More details about the experiment can be found in Refs. [4-10], while a complete discussion of the mathematical model is given in Ref. [13].

\section{A. Experiment}

The convecting fluid consists of a thin annular film of the liquid crystal material octylcyanobiphenyl (8CB), which is freely suspended between two concentric metal electrodes, as shown in Fig. 1. At the temperature of the experiment, the $8 \mathrm{CB}$ is in the smectic- $A$ phase in which the elongated liquid crystal molecules are aligned perpendicular to the plane of film and arranged in layers. Flow within the plane of the layers is isotropic and strongly 2D. The films consisted of an integer number of 20-100 smectic layers, each layer being $3.16 \mathrm{~nm}$ thick [16]. An applied electric voltage between the inner and outer electrodes drives an electric current through the film, which is doped to have a small ionic conductivity.

The electrical boundary conditions on the two free surfaces which separate the conducting film from charge-free space require that charges accumulate near these surfaces. The surface charge configuration is such that positive charges accumulate near the high electric potential at the inner electrode, while negative charges accumulate close to the grounded outer electrode. This inverted surface charge density is unstable to electric forcing in much the same way as the inverted mass density distribution of RBC is unstable to buoyancy forces. When the applied voltage $V$ exceeds the critical voltage $V_{c}$, convection sets in and the fluid is organized into cells in the form of vortices. A typical experimental procedure involves varying $V$, the main experimental control parameter, slowly from 0 to beyond $V_{c}$ in a sequence of small incremental steps past $V_{c}$ and then with decremental steps back to $0 \mathrm{~V}$. The quantitative measurements consist of current-voltage data; the total current $I$ flowing through the film under an imposed voltage $V$. The flow pattern is typically not visualized because suspending small particles in the film is difficult and tends to strongly perturb the conductivity and because particles aggregate due to their size being larger than the film thickness. In some experiments, an azimuthal flow could also be independently imposed by rotating the inner electrode. We do not consider this second control parameter in this paper.

The annular geometry of the film has a $Z_{2}$ reflection symmetry and is $\mathrm{O}(2)$ symmetric under continuous azimuthual rotations [17]. Below the onset of convection, the electrical forces are unable to overcome dissipation and the electric current is transported by a pure conduction mechanism that respects these underlying symmetries. At the primary bifurcation to convection at $V=V_{c}$, the film breaks the continuous symmetry under azimuthal rotations. The fluid is organized into laminar counter-rotating vortex pairs with a discrete mode number $m$, giving the flow a $D_{m}$ symmetry [17].

Without flow visualization, all that is experimentally observed is the total current $I$, which is equal to the conduction current $I_{0}$ only below the onset of convection. The dimensionless Nusselt number $\mathrm{Nu}=I / I_{0}$ characterizes the overall amplitude of convection. The reduced Nusselt number $\mathrm{Nu}$ -1 is a measure of the relative strength of the convective current to conducted current. The direct numerical simulation complements the experiment by allowing us to calculate $\mathrm{Nu}-1$, while also visualizing the dynamics of the complete velocity, electric potential, and charge density fields.

\section{B. Mathematical model}

In the experiment, the physical thickness of the film $s$ $\approx 0.1 \mu \mathrm{m}$ is much smaller than the width of the annulus $d$ $\approx 1 \mathrm{~mm}$. This, and the layered structure of the smectic, allows us to accurately model the film as a $2 \mathrm{D}$, Newtonian fluid confined to an annular space between infinitely thin electrodes which lie in the $x y$ plane. The rest of the threedimensional computational space is empty and free of charges. It can be shown [11] that the film is essentially surface dominated, as the ratio of bulk to surface forces on the film is $O(s / d)$. The constant thickness also means that the fluid is effectively incompressible. The fluid has 2D mass density $\rho$, shear viscosity $\eta$, and electrical conductivity $\sigma$.

The governing equations for $2 \mathrm{D}$ annular electroconvection are comprised of the mass, momentum, and charge conservation equations, with one additional Maxwell equation connecting the charge density $q$ and the electric potential $\psi$. Magnetic effects are negligible.

The governing equations are, in full [13],

$$
\begin{gathered}
\vec{\nabla} \cdot \overrightarrow{\mathbf{u}}=0, \\
\rho\left[\frac{\partial \overrightarrow{\mathbf{u}}}{\partial t}+(\overrightarrow{\mathbf{u}} \cdot \vec{\nabla}) \overrightarrow{\mathbf{u}}\right]=-\vec{\nabla} P+\eta \nabla^{2} \overrightarrow{\mathbf{u}}+q \overrightarrow{\mathbf{E}}, \\
\frac{\partial q}{\partial t}=-\vec{\nabla} \cdot(\sigma \overrightarrow{\mathbf{E}}+q \overrightarrow{\mathbf{u}}), \\
q=-\left.2 \varepsilon_{0} \partial_{z} \psi_{3}\right|_{z=0^{+}}, \\
\nabla_{3}^{2} \psi_{3}=\nabla_{2}^{2} \psi_{3}+\frac{\partial^{2} \psi_{3}}{\partial z^{2}}=0,
\end{gathered}
$$




$$
\psi_{2}=\psi_{3}(z=0)
$$

where $\overrightarrow{\mathbf{u}}$ is the fluid velocity, $P$ is the pressure, $q$ is the surface charge density, and $\overrightarrow{\mathbf{E}}=-\vec{\nabla}_{2} \psi_{2}$ is the electric field. Subscripts denote two- and three-dimensional potentials and gradients, and $\varepsilon_{0}$ is the permittivity of free space. We use cylindrical coordinates $(r, \theta, z)$.

The equation of mass conservation and the incompressibility condition yield a solenoidal velocity field $\overrightarrow{\mathbf{u}}=\left(u_{r} \hat{\mathbf{r}}+u_{\theta} \hat{\theta}\right)$ by Eq. (1). The flow velocity is determined from the conservation of momentum using Eq. (2), which is the 2D Navier-Stokes equations with an electrical body force $q \overrightarrow{\mathbf{E}}$. The conservation of charge is expressed by a continuity equation, Eq. (3), containing an ohmic conductive current density $\sigma \overrightarrow{\mathbf{E}}$ and a convective current density $q \overrightarrow{\mathbf{u}}$. Finally, the 2D charge density $q$ obeys Eq. (4), a Maxwell equation that describes the nonlocal relationship between the surface charge $q$ and the electric potential on the film $\psi_{2}$. The factor of 2 arises because the film has two free surfaces. Outside the film, there are no free charges, so the 3D electric potential $\psi_{3}$ obeys the Laplace equation, Eq. (4). Equation (6) expresses the fact that the electric potential is everywhere continuous; its value $\psi_{2}$ on the film acts as a boundary condition on the potential $\psi_{3}$ which fills the space outside the film.

The fluid velocity is subject to a nonslip boundary condition $u_{r}=u_{\theta}=0$ at the inner and outer radii of the annulus, $r=r_{i}$ and $r=r_{o}$, respectively. The potential $\psi_{2}$ is required to be $V$ on the inner electrode, $r \leq r_{i}$, and zero on the outer electrode, $r \geq r_{o}$. At infinity, $\psi_{3}=0$. The potential on the film itself can be found by specifying $q$ for $r_{i}<r<r_{o}$ and solving the mixed boundary value Laplace problem given by Eqs. (4) and (5) for $\psi_{3}$. Alternatively, $\psi_{2}$ can be specified on the film, and $\psi_{3}$ found self-consistently by solving the Dirichlet Laplace problem given by Eq. (5). The charge density $q$ then follows from Eq. (4). This nonlocal relationship between $q$ and $\psi$ is discussed in detail in the Appendix.

We employed the streamfunction-vorticity formulation for the primitive variables in the simulation. The streamfunction $\phi$ is given by $\overrightarrow{\mathbf{u}}=\vec{\nabla} \phi \times \hat{\mathbf{z}}$. In two dimensions, the vorticity $\omega$ is a scalar obeying $\vec{\nabla} \times \overrightarrow{\mathbf{u}}=\omega \hat{\mathbf{z}}$. In terms of the streamfunction $\phi$,

$$
\overrightarrow{\mathbf{u}}=u_{r} \hat{\mathbf{r}}+u_{\theta} \hat{\theta}=\frac{1}{r} \frac{\partial \phi}{\partial \theta} \hat{\mathbf{r}}-\frac{\partial \phi}{\partial r} \hat{\theta} .
$$

The advantages of using the stream-vorticity formulation are the elimination of the pressure $P$ and the replacement of the vector velocity by two simpler scalar fields, $\phi$ and $\omega$.

Starting from the stream-vorticity formulation, we rescaled length with the film width $d$, time with the charge relaxation time $\tau_{c}=\varepsilon_{0} d / \sigma$, and electric potential by the applied voltage $V$ at $r_{i}$. The dimensionless streamfunction $\phi$ and charge density $q$ are then scaled by $\sigma d / \varepsilon_{0}$ and $\varepsilon_{0} V / d$, respectively. We obtain the following dimensionless governing equations:

$$
\nabla^{2} \phi=-\omega
$$

$$
\begin{gathered}
\frac{\partial \omega}{\partial t}+(\overrightarrow{\mathbf{u}} \cdot \vec{\nabla}) \omega=\mathcal{P} \nabla^{2} \omega+\mathcal{P} \mathcal{R}\left(\vec{\nabla} \psi_{2} \times \vec{\nabla} q\right), \\
\frac{\partial q}{\partial t}+(\overrightarrow{\mathbf{u}} \cdot \vec{\nabla}) q=\nabla^{2} \psi_{2}, \\
\nabla_{3}^{2} \psi_{3}=0, \quad q=-\left.2 \partial_{z} \psi_{3}\right|_{z=0^{+}},
\end{gathered}
$$

where the important dimensionless parameters are

$$
\mathcal{R} \equiv \frac{\varepsilon_{0}^{2} V^{2}}{\sigma \eta} \quad \text { and } \quad \mathcal{P} \equiv \frac{\varepsilon_{0} \eta}{\rho \sigma d} .
$$

The main control parameter, the Rayleigh-like number $\mathcal{R}$, is a measure of the relative strength of applied electric forcing to viscous dissipation. The Prandtl-like number $\mathcal{P}$ is a fluid parameter which characterizes the ratio of the charge relaxation time to the viscous relaxation time. In addition, the geometry of the annulus is characterized by the radius ratio $\alpha \equiv r_{i} / r_{o}$. In dimensionless terms, the inner and outer radii are

$$
r_{i}=\frac{\alpha}{1-\alpha} \quad \text { and } \quad r_{o}=\frac{1}{1-\alpha} .
$$

The computational domain is the annulus $r_{i} \leq r \leq r_{o}$ and $0 \leq \theta<2 \pi$. We must also solve a Laplace equation for $\psi_{3}$ in the space $z \geq 0$. We decompose the solutions into an axisymmetric base state component (denoted by superscript 0 ) and a deviation from the base state solution (denoted by superscript 1) as follows:

$$
\begin{gathered}
\phi(r, \theta)=\phi^{(0)}(r)+\phi^{(1)}(r, \theta), \\
\omega(r, \theta)=\omega^{(0)}(r)+\omega^{(1)}(r, \theta), \\
q(r, \theta)=q^{(0)}(r)+q^{(1)}(r, \theta), \\
\psi_{2}(r, \theta)=\psi_{2}^{(0)}(r)+\psi_{2}^{(1)}(r, \theta), \\
\psi_{3}(r, \theta, z)=\psi_{3}^{(0)}(r, z)+\psi_{3}^{(1)}(r, \theta, z) .
\end{gathered}
$$

The charge and potential distributions in the base state, in which the fluid is quiescent, can be solved for analytically. Although the base state potential $\psi_{2}^{(0)}$ (given by Eq. 3.5 of Ref. [13]) is everywhere single valued and continuous, its $r$ derivatives are discontinuous at the edges of the film, where the potential on the film changes over to the constant imposed potential on the electrodes. This causes the base state charge density $q^{(0)}$, given analytically in terms of hypergeometric functions [13], to be divergent at the edges of the film. These divergences are integrable, however, and the overall base state is linearly unstable to the imposed electric force.

With the above decompositions, the unknowns to be computed are the deviations, which are solutions of the following set of equations:

$$
\begin{gathered}
\nabla^{2} \phi^{(1)}=-\omega^{(1)}, \\
\frac{\partial q^{(1)}}{\partial t}+J_{q, \phi}-\nabla^{2} \psi_{2}^{(1)}=0,
\end{gathered}
$$




$$
\begin{aligned}
& \frac{\partial \omega^{(1)}}{\partial t}+J_{\phi, \omega}=\mathcal{P} \nabla^{2} \omega^{(1)}+\mathcal{P} \mathcal{R} J_{\psi, q}, \\
& \nabla_{3}^{2} \psi_{3}^{(1)}=0, \quad q^{(1)}=-\left.2 \partial_{z} \psi_{3}^{(1)}\right|_{z=0},
\end{aligned}
$$

where $J_{q, \phi}, J_{\phi, \omega}$, and $J_{\psi, q}$ are the nonlinear Jacobian terms:

$$
\begin{aligned}
J_{q, \phi}= & \frac{1}{r}\left[\frac{\partial q^{(0)}}{\partial r} \frac{\partial \phi^{(1)}}{\partial \theta}+\frac{\partial q^{(1)}}{\partial r} \frac{\partial \phi^{(1)}}{\partial \theta}-\frac{\partial \phi^{(0)}}{\partial r} \frac{\partial q^{(1)}}{\partial \theta}\right. \\
& \left.-\frac{\partial \phi^{(1)}}{\partial r} \frac{\partial q^{(1)}}{\partial \theta}\right], \\
J_{\phi, \omega}= & \frac{1}{r}\left[\frac{\partial \omega^{(0)}}{\partial r} \frac{\partial \phi^{(1)}}{\partial \theta}+\frac{\partial \omega^{(1)}}{\partial r} \frac{\partial \phi^{(1)}}{\partial \theta}-\frac{\partial \phi^{(0)}}{\partial r} \frac{\partial \omega^{(1)}}{\partial \theta}\right. \\
& \left.-\frac{\partial \phi^{(1)}}{\partial r} \frac{\partial \omega^{(1)}}{\partial \theta}\right], \\
J_{\psi, q}= & \frac{1}{r}\left[\frac{\partial \psi_{2}^{(0)}}{\partial r} \frac{\partial q^{(1)}}{\partial \theta}-\frac{\partial q^{(0)}}{\partial r} \frac{\partial \psi_{2}^{(1)}}{\partial \theta}+\frac{\partial \psi_{2}^{(1)}}{\partial r} \frac{\partial q^{(1)}}{\partial \theta}\right. \\
& \left.-\frac{\partial q^{(1)}}{\partial r} \frac{\partial \psi_{2}^{(1)}}{\partial \theta}\right] .
\end{aligned}
$$

The variables $\phi^{(1)}, \psi_{2}^{(1)}$, and $\psi_{3}^{(1)}$ satisfy the following boundary conditions for $r=r_{o}$ and $r_{i}$ :

$$
\begin{gathered}
\phi^{(1)}(\theta)=\partial_{r} \phi^{(1)}(\theta)=\psi_{2}^{(1)}(\theta)=0, \\
\psi_{3}^{(1)}(r, \theta, z=0)= \begin{cases}0 & 0 \leq r \leq r_{i}, \\
\psi_{2}^{(1)}(r, \theta) & r_{i} \leq r \leq r_{o}, \\
0 & r \geq r_{o} .\end{cases}
\end{gathered}
$$

The Jacobians $J_{q, \phi}$ and $J_{\psi, q}$ each contain terms proportional to $\partial q^{(0)} / \partial r$, which diverges at the edges of the film. Fortunately, in each case, these terms multiply quantities that go to zero at the edges of the film and the overall expressions remain finite. Similarly, the piecewise continuous nature of the boundary conditions on $\psi_{3}^{(1)}$, given by Eq. (22), implies that $\partial q^{(1)} / \partial r$ diverges at the edges of the film, but in Eqs. (18) and (20), these divergences multiply quantities that go to zero.

We now turn to the numerical solution of these equations.

\section{DIRECT NUMERICAL SIMULATION}

We constructed a time-stepping, pseudospectral code to calculate the solutions for the perturbations governed by Eqs. (14)-(17). We compared two different time-discretization schemes in order to check the accuracy of the solutions. We then used the solutions to calculate some integrated physical quantities which could be related directly to experiment.

\section{A. Time-discretization methods}

The first time-discretization method we used was the Adams-Bashforth and backward-differentiation (AB/BDI2) scheme [18]. In this method, the time derivative was mod- eled by $\partial_{t} U \approx\left(3 U^{k+1}-4 U^{k}+U^{k-1}\right) /(2 \Delta t)$, where the superscript $k$ denotes the time-stepping index and $\Delta t$ was a properly chosen discrete time step size. The diffusion term $f$ was approximated by $f^{k+1}$, using a backward scheme. The nonlinear Jacobian terms and the external forcing terms $F$ were estimated with the first order Adams-Bashforth scheme: $\mathrm{AB}_{1}\{F\}=2 F^{k}-F^{k-1}$. The combination of these two first-order approximation schemes, Adams-Bashforth and backward differentiation, gives the discretized equations to second order accuracy.

We also used the semi-implicit first order Euler differentiation scheme [19] as a second method of time discretization. In this method, the time derivative is approximated by $\partial_{t} U \approx\left(U^{k+1}-U^{k}\right) /(\Delta t)$. The nonlinear terms and external forcing terms were estimated by the forward Euler scheme, i.e., using the values $F^{k}$ at current time step $k$, while the diffusion term was approximated by $f^{k+1}$, using a backward Euler scheme.

\section{B. Numerical solution of the time-discretized equations}

We solved the time-discretized equations using a pseudospectral method. The streamfunction $\phi$, the vorticity $\omega$, the 2D electric potential $\psi_{2}$, and the surface charge density $q$ were approximated by a truncated Fourier series in the $\hat{\theta}$ direction and by Chebyshev polynomials in the $\hat{r}$ direction,

$$
\begin{aligned}
& \phi^{(1)}(r, \theta, t)=\sum_{m=-K}^{K} \hat{\phi}_{m}(r, t) e^{i m \theta}, \\
& \omega^{(1)}(r, \theta, t)=\sum_{m=-K}^{K} \hat{w}_{m}(r, t) e^{i m \theta}, \\
& \psi_{2}^{(1)}(r, \theta, t)=\sum_{m=-K} \hat{\psi}_{2 m}(r, t) e^{i m \theta}, \\
& q^{(1)}(r, \theta, t)=\sum_{m=-K}^{K} \hat{q}_{m}(r, t) e^{i m \theta} .
\end{aligned}
$$

The variables $\phi, \omega, \psi_{2}$, and $q$ are all real so in practice we solve for $m=0,1, \ldots, K$ and use the complex conjugate relationship $\hat{\phi}_{-m}=\hat{\phi}_{m}^{*}$ for $m<0$.

The 2D electric potential $\psi_{2}$ and the surface charge density $q$ are not freely evolving, independent variables. In fact, they evolve simultaneously so that at each time step they are linked by the nonlocal coupling described in Sec. II B. In the Appendix, we describe how we numerically calculated the nonlocal relationship between $\hat{\psi}_{2 m}(r)$ and $\hat{q}_{m}(r)$ by solving Eqs. (4) and (5). This instantaneous relationship can be computed in a separate calculation and then applied at each time step, which greatly simplifies the time stepping procedure.

The partial differential equations given by Eqs. (14)-(17) were converted into ordinary differential equations (ODEs) in $r$ by substituting Eqs. (23)-(26) and using the orthogonality of the Fourier modes. We then employed the Chebyshev collocation method $[20,21]$ to solve the ODEs with the Fou- 
rier coefficients as unknowns. There were $N_{c}+1$ grid points in the radial direction, where $N_{c}$ is the order of the highest Chebyshev polynomial included. The radial range from $r_{i}$ to $r_{o}$ is linearly mapped onto a new variable $x$ using

$$
x=2 r-\left[\frac{1+\alpha}{1-\alpha}\right],
$$

such that $-1 \leq x \leq 1$ spans the film. The collocation method approximates the solution as a truncated Chebyshev polynomial series and makes the residuals at collocation points $x_{j}$ $=\cos \left(\pi j / N_{c}\right), j=0,1,2, \ldots, N_{c}$ equal to zero. The unknowns are then the Fourier spectral values of the variables of interest at the $N_{c}+1$ collocation points, i.e., $\hat{\phi}^{k}\left(x_{j}\right), \hat{\omega}^{k}\left(x_{j}\right)$, and $\hat{\psi}_{2}^{k}\left(x_{j}\right)$, for $j=0,1, \ldots, N_{c}$, and at each time step $k$.

The nonlinear terms in Eqs. (14)-(17) were calculated using the pseudospectral technique [20] which consists of performing the differentiations in the spectral space and the products in the physical space. The spectral space and physical space are connected computationally by a fast Fourier transform.

We investigated the primary bifurcation to convection as a function of the Rayleigh-like control parameter $\mathcal{R}$, the radius-ratio $\alpha$, and the Prandtl-like number $\mathcal{P}$. We ran the time stepping simulation by gradually increasing $\mathcal{R}$ in small increments separated by many time steps, starting from the conduction state, passing through the onset of convection at $\mathcal{R}_{c}$. For $\mathcal{R}<\mathcal{R}_{c}$, we used initial conditions such that $\phi=0$, so that the fluid was quiescent. The electric potential $\psi_{2}$ was given by random white noise with amplitude in the range $10^{-5}-10^{-4}$. For $\mathcal{R}>\mathcal{R}_{c}$, we used the converged, steady-state numerical solution at the previously calculated $\mathcal{R}$ as the initial condition for the next $\mathcal{R}$. The radial boundary conditions applied to the Fourier coefficients for all modes $m$ were

$$
\hat{\phi}_{m}=\frac{d}{d r} \hat{\phi}_{m}=\hat{\psi}_{2 m}=0 \quad \text { for } \quad r=r_{o}, r_{i} .
$$

To enforce the rigid boundary condition on the streamfunction $\phi$ in Eq. (28), we used the influence matrix method $[19,20]$ to calculate the corresponding Dirichlet boundary condition on the vorticity $\omega$. The Jacobian terms in Eqs. (15) and (16) were computed in the Fourier space with a "3/2rule" antialiasing technique [20].

Overall, the method described above is extremely conservative and stable, yet is still efficient enough that we can reach $\mathcal{R} \sim 1000$ with only modest computational effort.

\section{Integrated physical quantities of interest}

The electric Nusselt number $\mathrm{Nu}$ is a dimensionless measure of the fraction of the total current transported by convection. It is the electrical analog of the thermal Nusselt number which characterizes the total heat transport in Rayleigh-Bénard convection [1].

$\mathrm{Nu}$ is defined to be the ratio of the total current to the conductive current. The azimuthal components of the current density average to zero around the annulus, leaving only the radial contribution, which can be integrated to give

$$
\mathrm{Nu}=\frac{\int_{0}^{2 \pi}\left(u_{r} q-\partial_{r} \psi_{2}\right) r d \theta}{\int_{0}^{2 \pi}\left(-\partial_{r} \psi_{2}^{(0)}\right) r d \theta} .
$$

Taking advantage of the zero radial velocity at the boundaries to simplify Eq. (29), Nu can be computed numerically from

$$
\mathrm{Nu}=1+\left[r \log (\alpha) \frac{d}{d r} \hat{\psi}_{2 m=0}^{(1)}(r)\right]_{r=r_{i}} .
$$

The term in the square brackets can be taken at either $r_{i}$ or $r_{o}$. $\mathrm{Nu}$ is directly related to the physical currents that can be measured experimentally [5-10]. It is also a direct measure of the amplitude of the convective velocity.

We also calculated the mean area density of the kinetic energy

$$
E_{\text {kin }}=\frac{1}{2 a} \int_{r_{i}}^{r_{o}} \int_{0}^{2 \pi} \overrightarrow{\mathbf{u}} \cdot \overrightarrow{\mathbf{u}} r d r d \theta,
$$

where $a$ is the dimensionless area of the annulus. $E_{\text {kin }}$ is a useful diagnostic of the strength of convection.

To test the convergence of the time stepping code onto a steady state solution, we calculated the changes in $\mathrm{Nu}$ and $E_{\text {kin }}$, as well as the norm of the change in Fourier coefficients between one time step and the previous one. The solutions were considered converged not only when the norm was less than $10^{-5}$ but also when the changes in $\mathrm{Nu}$ and $E_{\text {kin }}$ were $\leq 0.05 \%$.

\section{RESULTS AND DISCUSSION}

We first validated the code by simulating weakly forced conditions near the onset of convection. The quantitative numerical results for the critical Rayleigh-like number $\mathcal{R}_{c}$ at onset were benchmarked and found to be in better than $2 \%$ agreement with the predictions of linear stability theory [13].

The annular geometry, described by the radius ratio $\alpha$, has various interesting effects on $\mathcal{R}_{c}$ and the critical number of vortex pairs $m_{c}$ at the onset of convection, both of which are predicted by linear theory [13] and measured experimentally [6]. In general, the main effect of varying $\alpha$ is to select the overall azimuthal mode, which is quantized to fit an integer number of vortex pairs around the perimeter of the annular cell. For $\mathcal{R}>\mathcal{R}_{c}$, nonlinear effects make themselves felt. The annulus has a reflection symmetry and a continuous symmetry under azimuthal rotations. We can deduce something about the nonlinear state from these symmetries alone. We expect, on the basis of these symmetries, that a generic Landau amplitude equation will describe the neighborhood of the primary bifurcation in the weakly nonlinear regime $[2,6]$. For a steady convective state, the time independent amplitude equation is [6]

$$
\varepsilon A-g A^{3}-h A^{5}+f=0,
$$

where $A$ is the amplitude of convection and $\varepsilon=\left(\mathcal{R} / \mathcal{R}_{c}\right)-1$ is the reduced control parameter. The coefficient $g$ of the cubic nonlinear term determines whether the bifurcation to electroconvection is continuous (supercritical) for $g>0$, discontinu- 

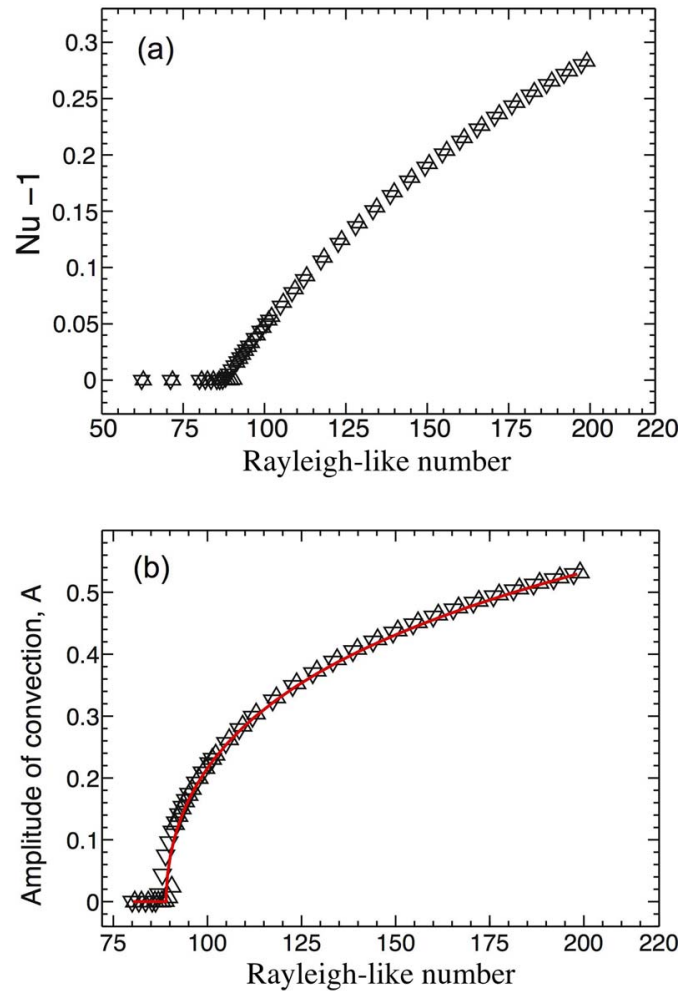

FIG. 2. (Color online) Representative numerical data for the dimensionless convective current, $\mathrm{Nu}-1$, as the Rayleigh-like number $\mathcal{R}$ changes. Here the other parameters are $\alpha=0.56$ and $\mathcal{P}=10$. Data obtained for increasing (decreasing) $\mathcal{R}$ are shown as $\triangle(\nabla)$. (b) The corresponding amplitude of convection $A=\sqrt{\mathrm{Nu}-1}$ as a function of $\mathcal{R}$. The solid line is a nonlinear least-squares fit of the data to the Landau amplitude equation given by Eq. (32).

ous (subcritical) for $g<0$, or tricritical for $g=0$. The field term $f$ allows for some imperfection in the bifurcation and was found to be necessary to realistically model small symmetry-breaking imperfections in the real laboratory experiment [6]. The amplitude $A$ can be scaled to the reduced Nusselt number [12] so that $A^{2}=\mathrm{Nu}-1$. Mimicking the analysis used previously on real experimental data [6], we determined $\mathcal{R}_{c}, g, h$, and $f$ by fitting the numerical data for $\mathrm{Nu}-\mathcal{R}$ to Eq. (32) using a nonlinear least-squares method. We could then numerically investigate the dependence of $\mathcal{R}_{c}$, $m_{c}$, and $g$ over a broad parameter space of radius ratio $\alpha$ and Prandtl-like number $\mathcal{P}$. We are able to reach a wider range of these parameters than was possible in previous theoretical and experimental work.

\section{A. Dependence on the Rayleigh-like number $\mathcal{R}$}

Using the numerical model, we follow the experimental protocol of ramping $\mathcal{R}$ slowly up and down through onset. Experimentally, this is done by increasing and decreasing the applied voltage.

Figure 2(a) shows the dimensionless current carried by convection, $\mathrm{Nu}-1$, as the control parameter $\mathcal{R}$ varies. Zero convective current indicates the conduction regime in which dissipation effects dominate and prevent convection even under the electric forcing. Slowly increasing $\mathcal{R}$, we observed a

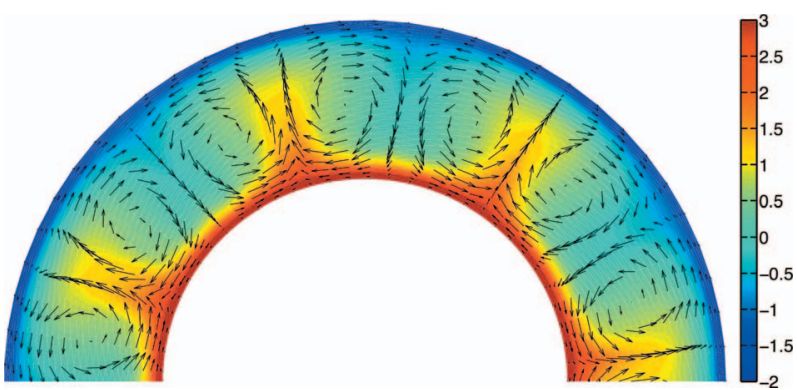

FIG. 3. (Color) The basic fields for steady convection at $\mathcal{R}=199.8, \alpha=0.56$, and $\mathcal{P}=10$ : The total electric charge density $q$ (color) and the velocity field (black). Only the upper half of the annular cell is shown. Positive charge moves away from the high potential electrode at the inner radius, while negative charge moves away from the grounded outer electrode.

critical threshold showing the onset of convection at $\mathcal{R}_{c}$. Near this bifurcation, we observed critical slowing down, indicated by extremely long convergence times. We sometimes observed a slight hysteresis due to dynamical effects. It is well known both analytically and experimentally that a bifurcation point is shifted when a control parameter is swept through a bifurcation at a finite rate [22]. We allow for critical slowing down by greatly increasing the computational time allotted to reach the convergence criterion. However, a small residual delay in the bifurcation with increasing $\mathcal{R}$ is still observable in Fig. 2(b), which shows the amplitude of convection $A$ vs $\mathcal{R}$.

Above $\mathcal{R}_{c}$, a pattern of stationary convective vortices is formed that carries extra current and breaks the continuous symmetry under azimuthal rotation. Convection remains steady in the weakly nonlinear regime up to $\mathcal{R} \sim 5 \mathcal{R}_{c}$. Figure 3 shows the surface charge distribution and the corresponding velocity field for steady convection in which the laminar flow provides a constant contribution to the electric current.

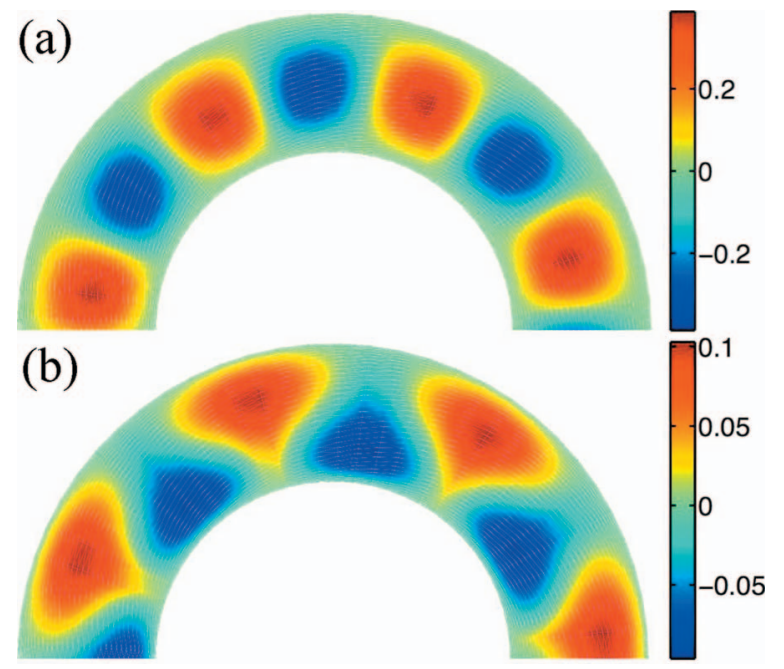

FIG. 4. (Color) (a) The streamfunction and (b) the perturbed 2D electric potential $\psi_{2}^{(1)}$ for the same control parameters as in Fig. 3. For this specific $\alpha$, the state is dominated by the $m=7$ Fourier mode and there are seven counter-rotating vortex pairs. 

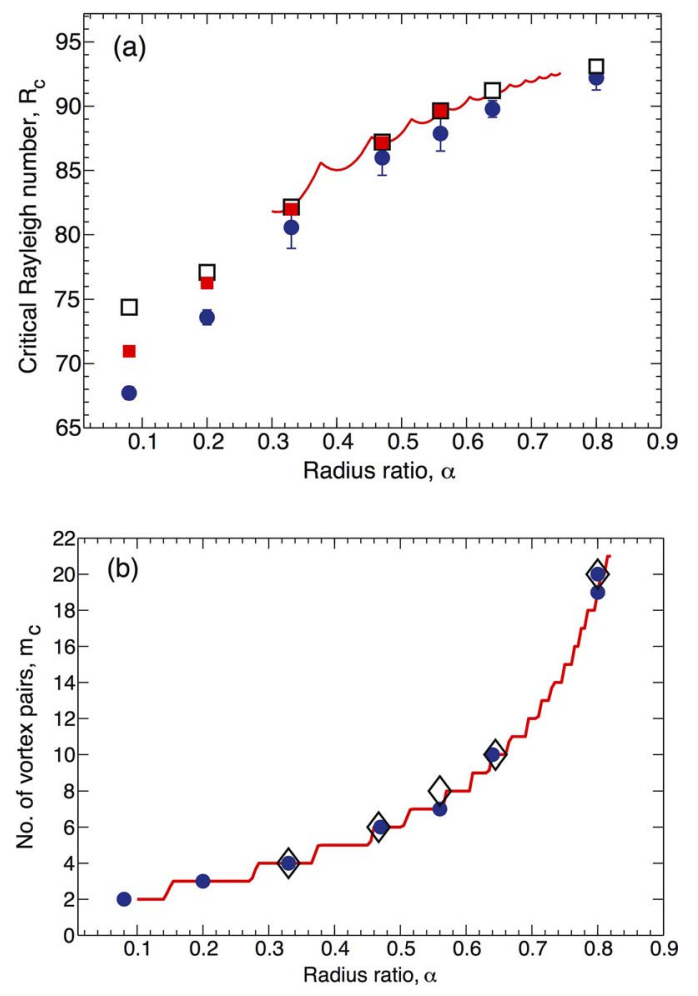

FIG. 5. (Color online) (a) The radius ratio dependence of the critical Rayleigh number $\mathcal{R}_{c}$. The solid circles $(\bullet)$ are the results of the numerical simulation. Open and filled boxes $(\square, \boldsymbol{\square})$ are the theoretical predictions of nonlocal linear stability analysis, using third order and sixth order expansions in the radial direction, respectively. (b) The critical number of counter-rotating vortex pairs $m_{c}$. All the simulation data $(\bullet)$ are for a fixed $\mathcal{P}=10$. The solid lines are the predictions of fully nonlocal linear stability theory and $\diamond$ are experimental results from Ref. [6].

The corresponding streamfunction and perturbed electric potential are shown in Fig. 4. From these figures, we see narrow positively charged regions which are being carried by the flow toward the grounded outer electrode, separated by broader negatively charged regions returning. These local convective currents add to the total current and act to reduce the applied potential gradient. The simulation provides insightful visualizations of the local fields that nicely complement the physical experiments which could not be visualized. This insight will play an important role in understanding more complicated bifurcations at higher $\mathcal{R}$, eventually into the turbulent regime $[9,10]$, and the complex rotating states that occur under an applied shear [6-8].

\section{B. Dependence on geometry}

The radius ratio $\alpha=r_{i} / r_{o}$ strongly influences the critical Rayleigh-like number $\mathcal{R}_{c}$, the critical mode number $m_{c}$, and, to a lesser extent, the nonlinear saturation coefficient $g$.

Figure 5(a) shows the $\alpha$ dependence of $\mathcal{R}_{c}$ from numerical computations and from linear stability theory [13]. The trend of $\mathcal{R}_{c}$ in the numerical data is increasing overall as a function of the radius ratio and quantitatively agrees with a

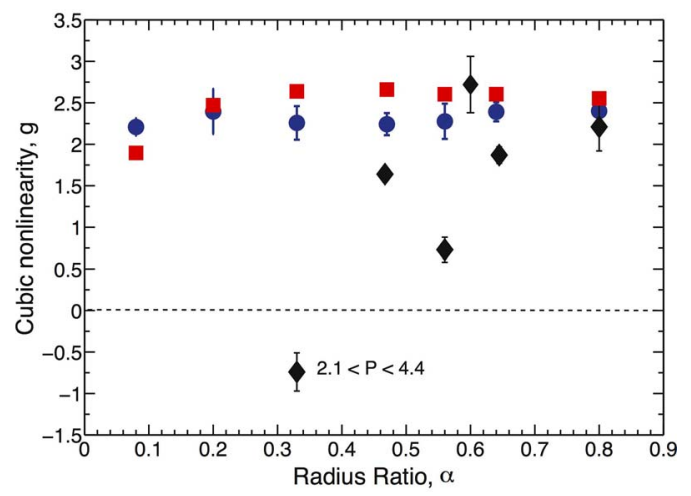

FIG. 6. (Color online) The radius ratio dependence of the coefficient of the cubic nonlinearity $g$. Numerical data $(\bullet)$ for $\mathcal{P}=10$ with various radius ratios $\alpha$ show a continuous and supercritical bifurcation $(g>0)$. They agree well with a nonlocal theory prediction for $\mathcal{P}=123$ shown by red filled squares $(\boldsymbol{\square})$. Black filled diamonds $(\diamond)$ are experimental results, from Ref. [6], for various $\mathcal{P}$ $>1$.

fully nonlocal theory calculation shown by the solid line [13].

We used several values of $N_{c}$ and $K$, ranging between 29 and 45 and between 32 and 64, respectively. Numerical data with different values of $N_{c}$ and $K$ agree with each other to within a small scatter. The error bars in Fig. 5(a) were obtained from the spread of numerical data and were calculated using different time step sizes, random initial conditions, grid sizes, and time discretization approximations. Each data point consists of at least three complete sets of numerical runs, sweeping the voltage up and down.

The calculation becomes more difficult for small $\alpha$, due to the increasing asymmetry between the inner and outer electrodes. We were able to numerically explore with reasonable accuracy down to $\alpha=0.1$. This is well below the minimum radius reached experimentally, which corresponded to $\alpha$ $=0.33$. Various truncations also limit the range of the nonlocal linear stability theory [13] to $0.33 \leq \alpha \leq 0.8$. Although its accuracy is somewhat compromised, the numerical code remains very stable and can thus be used to broaden the range of $\alpha$ accessible. At the smallest $\alpha, m_{c}=2$ and there are just four vortices around the annulus.

Figure 5(b) shows critical mode number $m_{c}$ from the simulation, linear theory, and experiment [13]. For special values of $\alpha$, two adjacent values of azimuthal mode number are simultaneously unstable at onset and $\mathcal{R}_{c}(\alpha)$ exhibits a cusp, giving it a scalloped structure. These are the special CoD2 points which we discuss in detail in Sec. IV D below.

Numerical simulations reveal that the primary bifurcation is continuous and supercritical for $\mathcal{P}=10$ and across a broad range of $\alpha$, as shown in Fig. 6. Values of $g$ are found between 2.0 and 2.6. These values are in excellent agreement with calculations of $g$ for $0.60 \leq \alpha \leq 0.80$ from a local, weakly nonlinear theory [8]. Both numerical and theoretical data therefore suggest only a weak dependence of $g$ on $\alpha$.

These results can be compared to experimental measurements in which $g$ was extracted by fitting current-voltage data [6]. The experimental results, which show considerable scatter, are also shown in Fig. 6. The experiment generally 


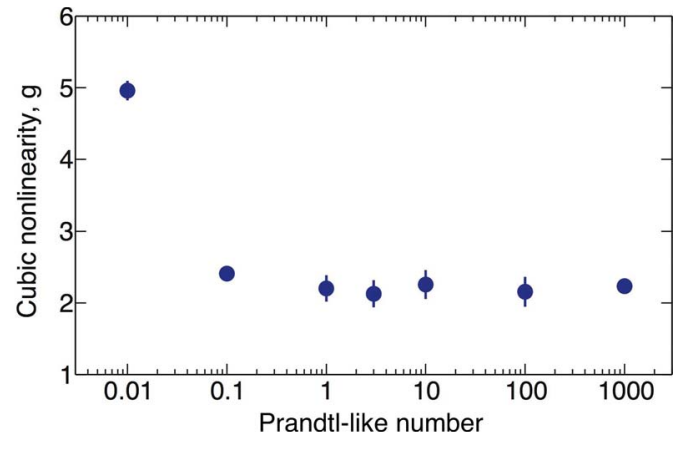

FIG. 7. (Color online) Simulation data of the cubic nonlinearity coefficient $g$ over a wide range of $\mathcal{P}$ for a fixed $\alpha=0.33$. The numerical data show supercritical bifurcations $g>0$ for various $\mathcal{P}$ and the $\mathcal{P}$ independence of $g$ for $\mathcal{P}>0.1$.

shows supercritical bifurcations for various $\mathcal{P}>1$. For the larger $\alpha$, these agree with simulation and weakly nonlinear theory.

For small $\alpha$, and particularly for the smallest $\alpha=0.33$, a systematic disagreement is observed. At $\alpha=0.33$, the experimentally measured $g$ actually becomes negative, indicating a subcritical bifurcation which is in clear disagreement with the simulation and weakly nonlinear theory. The most likely explanation for this disagreement is that the geometry of the electrodes in the experimental cell deviates more from the idealizations of the model as $\alpha$ becomes smaller. The support structure for the center electrode may become significant. We observe that the field term $f$ in the fitting function Eq. (32), which describes the imperfection of the bifurcation, increases for small $\alpha$. Also, the result for $g$ at $\alpha=0.33$ was obtained by averaging over data taken over a range of $\mathcal{P}$, which may have introduced some bias since the experimental values of $\mathcal{P}$ tend to be closer to one, and therefore fractionally more uncertain, for small $\alpha$. More experiments will be required to sort out this discrepancy.

\section{Dependence on the Prandtl-like number $\mathcal{P}$}

The Prandtl-like number $\mathcal{P}$ measures the dimensionless ratio of the charge and viscous relaxation times. Large $\mathcal{P}$ limits the influence of the nonlinear advection and time derivative terms compared to the viscous and external driving force terms. Linear stability analysis predicts that $\mathcal{R}_{c}$ should be independent of $\mathcal{P}$ for all $\alpha$. This result follows from the fact that the base state is quiescent. In particular, linear theory predicts $\mathcal{R}_{c} \approx 82$ at $\alpha=0.33$. In agreement with this, the simulation data at $\alpha=0.33$ consistently shows values of $\mathcal{R}_{c}$ spread between 80 and 82 for a wide range of $\mathcal{P}$ between 0.01 and 1000 .

The Prandtl-like number $\mathcal{P}$ has an effect on the nonlinear behavior, and in particular on the coefficient of the cubic nonlinearity $g$. Figure 7 shows $g$ for a range of $\mathcal{P}$ between 0.01 and 1000 . This is a much wider range than that achievable experimentally. The smallest value achieved in experiments was $\mathcal{P} \sim 2$. The simulation data for $\alpha=0.33$ show very little dependence on $\mathcal{P}$ for $0.1 \leq \mathcal{P} \leq 1000$. However, for small $\mathcal{P}<0.01, g$ increases by a factor of $\gtrsim 2$. This is a dramatic change compared to the near independence of $g$ on
$\mathcal{P}$ for $0.1 \leq \mathcal{P} \leq 1000$. The simulation data agree with previous theoretical calculations [8]. Experimental results also show the $\mathcal{P}$ independence of $g$ for large $\mathcal{P}$. For example, $g$ $\approx 2$ for $25<\mathcal{P}<65$ and $\alpha=0.64$ [6].

\section{Codimension-two points}

Numerous codimension-two (CoD2) points exist in the parameter space of the Rayleigh-like number $\mathcal{R}$ and the radius ratio $\alpha$. At such CoD2 points, two adjacent azimuthal modes, $m$ and $m+1$, become simultaneously unstable at onset. This allows for unusual mode interactions near such points, which may lead to complex dynamics close to onset. The location of CoD2 points can be predicted by linear stability analysis. They appear in Fig. 5(a) at the intersection points of the scallop-shaped curves which map the onset values of $\mathcal{R}$ for each $m$. The dynamics of mode competition close to these CoD2 point has not previously been studied experimentally, or by weakly nonlinear analysis. The present numerical simulation gives us the tools to investigate the dynamics near onset, close to a CoD2 point.

Langford and Rusu previously studied patterns in annular electroconvection using equivariant bifurcation theory [17]. They enumerated the possible bifurcations near CoD2 points. Under the assumption of a supercritical primary bifurcation, they predicted only two possible scenarios for the low lying secondary bifurcations. In one case, there is a smooth transition from the $m$ to the $m+1$ mode via a stable mixed-mode branch of $Z_{2}$ symmetry. This preserves reflection symmetry of the annulus while breaking the azimuthal rotational symmetry. Under the other scenario, there is a hysteretic jump between the stable branches for modes $m$ and $m+1$.

We selected $\alpha=0.452$, which is predicted by linear theory [13] to be close to the CoD2 point for $m=5$ and $m=6$. Close to this $\alpha$, for $\mathcal{R}$ slightly larger than $\mathcal{R}_{c}$, the numerical simulation shows that the amplitudes of the $m=5$ and $m=6$ Fourier modes both show positive growth for a long period of time, up to $\approx 150 \tau_{c}$. However, the competition between the two modes is such that one mode eventually decays while the other saturates to a steady state after a long time. Figure 8 shows the complete evolution. Figure 9 shows the end result of the mode competition at $\alpha=0.452$, for two slightly different values of $\mathcal{R}$ and different random white noise initial conditions. The final state is very sensitive to the initial condition, and it is found to latch into either $m=5$ or $m=6$.

The numerical simulation thus shows that, at least for this case, the secondary bifurcation is of the hysteretic type, corresponding to Fig. 3a in Ref. [17]. It is nevertheless possible to observe a mixed mode. Figure 8(a) shows the streamfunction of the coexisting $m=5$ and $m=6$ state, which does have the expected $Z_{2}$ symmetry. However, this mixed-mode state is transient, unstable, and eventually relaxes to a single-mode state. At present, we know of no CoD2 points which show stable mixed modes, although not all values of $\alpha, \mathcal{R}$, and $\mathcal{P}$ have been explored.

\section{CONCLUSION}

In this paper, we have employed direct numerical simulation to analyze electrically driven convection in an annular 

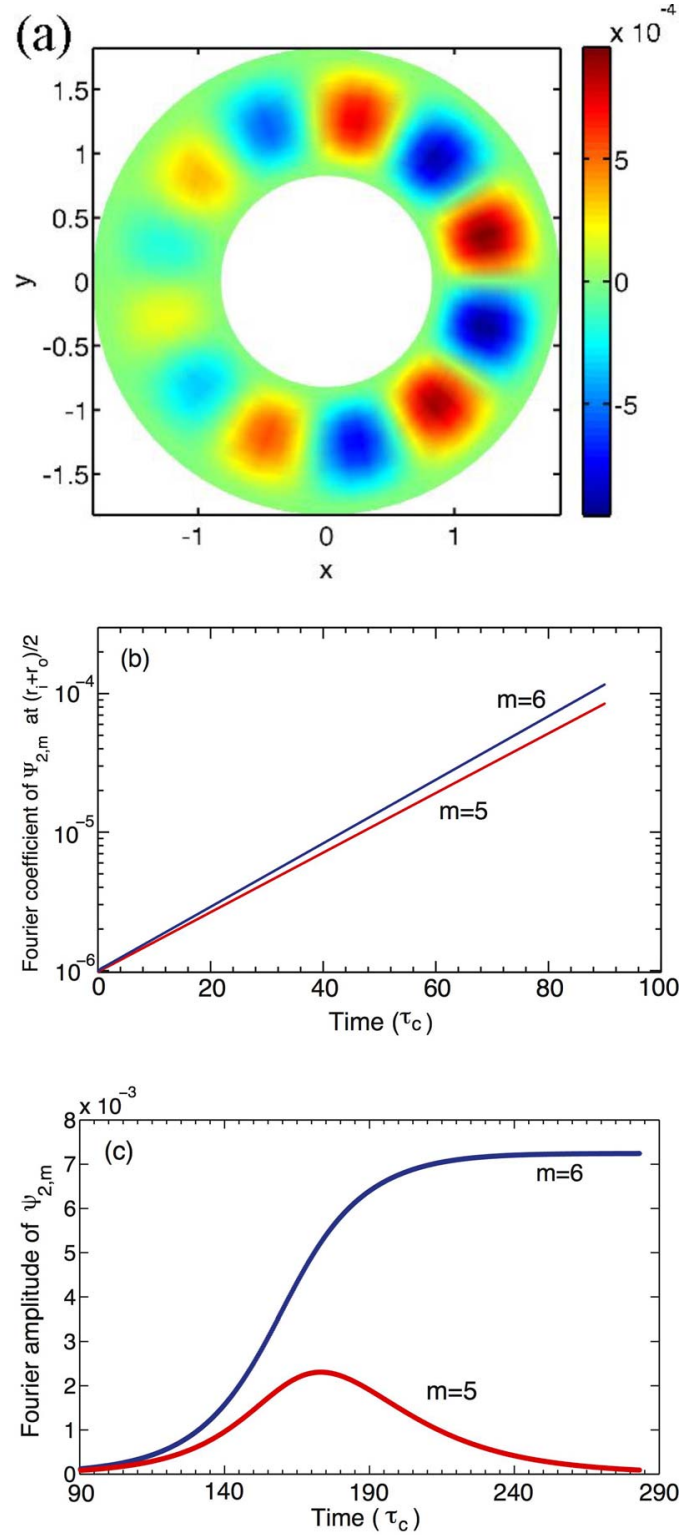

FIG. 8. (Color online) Mode competition close to the CoD2 point at $\alpha=0.452$ and $\mathcal{R}=87$. (a) The streamfunction of a transient mixed-mode state with $m=5$ and $m=6$ components. This state corresponds to the $Z_{2}$ symmetric solution predicted in Ref. [17]. (b) The early time growth of the Fourier amplitudes of modes $m=5$ and $m=6$, starting from an initial condition of equally large amplitudes for both modes. The growth rates of $\hat{\psi}_{2 m}$ are both $\approx 0.05$, for early times $\$ 90 \tau_{c}$. (c) The full time evolution of the amplitudes of the two modes, showing that mode competition eventually results in one mode suppressing the other.

thin film. We computed the critical Rayleigh-like number $\mathcal{R}_{c}$, critical mode number $m_{c}$, and the coefficient $g$ of the cubic term in the Landau amplitude equation as a function of the radius ratio $\alpha$, a parameter that completely characterizes the bounded annular geometry. We also found the variation of the coefficient $g$ with the Prandtl-like number $\mathcal{P}$ for one fixed value of $\alpha$. The numerical results are generally in good agreement with experimental data and previous theoretical studies based on linear stability [13] and weakly nonlinear analysis. We have established that the primary bifurcation at onset is supercritical for a wide range of $\alpha$ and $\mathcal{P}$. The only disagreement with experimental results, which is so far unexplained, comes for small $\alpha$, for which fits to experimental data indicated a weakly backward primary bifurcation.

These calculations nicely complement previous experimental studies, for which no visualization was possible, by allowing us to image the various fields in space and time, as well as to extend significantly the range of $\alpha$ and $\mathcal{P}$ that can be reached. The visualization and detailed numerical studies revealed the dynamics near a codimension-two point. The competition between critical modes $m$ and $m+1$ proceeds via a mixed state that possesses $Z_{2}$ reflection symmetry, as first suggested by equivariant bifurcation theory [17].

This work may be extended in several interesting directions. In future work, we hope to simulate the effect of superposing an azimuthal shear flow on the convection. Experiments and linear theory [13] of this situation have shown that the shear suppresses convection and leads to a rich variety of bifurcation scenarios. In addition, we plan to push the simulation to the high Rayleigh-like number regime, in which scaling has been observed experimentally $[9,10]$.

\section{ACKNOWLEDGMENTS}

We gratefully thank the Isaac Newton Institute for Mathematical Sciences for support, and the Canadian Institute for Theoretical Astrophysics (CITA) for access to computational facilities. This research was supported by the Natural Sciences and Engineering Research Council of Canada.

\section{APPENDIX: RELATIONSHIP BETWEEN SURFACE CHARGE AND 2D ELECTRIC POTENTIAL}

The surface charge density $q$ and the 2D electric potential on the film $\psi_{2}$ are nonlocally related. Either of these two quantities, plus the 2D potential on the electrodes, form the boundary conditions for the 3D Laplace equation that determines the potential outside the film. The remaining 2D quantity must be determined self-consistently from the solution of the 3D Laplace problem. This nonlocal relationship makes the simulation of electroconvection more complicated than that of thermally driven RBC. This additional complication is evident by the coupling of four unknown quantities in the governing equations, instead of the usual three for RBC. Fortunately, the additional potential and charge fields are coupled instantaneously by a Maxwell equation, so that the nonlocal calculation involves no additional time derivatives, and can thus be solved once and for all before time stepping the other fields. This calculation must ultimately be done numerically, but the Laplace problem can first be solved implicitly in integral form. The calculation is made more straightforward by the decomposition of the fields into Fourier modes in the pseudospectral technique.

Since no free charge exists outside of the film, the 3D potential satisfies the Laplace equation $\nabla^{2} \psi_{3}=0$ with appropriate boundary conditions (BCs) on the film and electrodes, which fill the $x y$ plane. The surface charge density $q$ is determined by the discontinuity in $\psi_{3}$ in the $\hat{\mathbf{z}}$ direction on the 

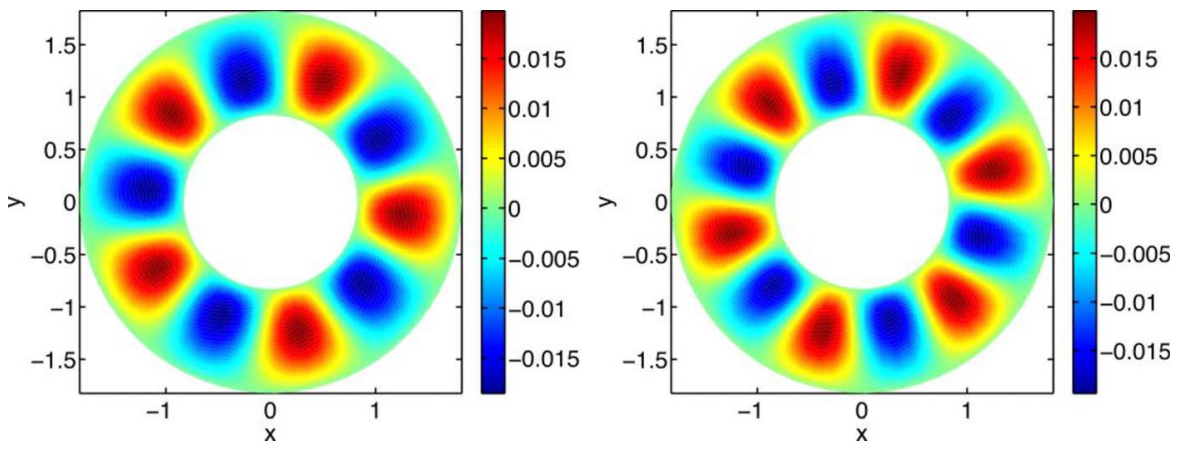

FIG. 9. (Color online) Perturbed 2D electric potential $\psi_{2}$ near a codimension-two point (CoD2) at $\alpha=0.452$. The Rayleigh-like numbers were $\mathcal{R}$ $=87.006$ for $m=5$ (left) and $\mathcal{R}$ $=87.859$ for $m=6$ (right). Both patterns used different random white noise as the initial condition. surface of the film. The BCs require that $\psi_{3}$ in charge-free space is finite for $z \geq 0$ and vanishes for $z \rightarrow \infty$. General solutions for $z \geq 0$ are

$$
\begin{gathered}
\psi_{3}(r, \theta, z)=\sum_{m=-\infty}^{\infty} \int_{0}^{\infty} e^{-k z} J_{m}(k r) A_{m}(k) e^{i m \theta} d k, \\
q(r, \theta)=-\left.2 \partial_{z} \psi_{3}\right|_{z=0^{+}} \\
=\left.2 \sum_{m=-\infty}^{\infty} \int_{0}^{\infty} k e^{-k z} J_{m}(k r) A_{m}(k) e^{i m \theta} d k\right|_{z=0^{+}} \\
=\sum_{m=-\infty}^{\infty} \hat{q}_{m}(r) e^{i m \theta} \approx \sum_{m=-K}^{K} \hat{q}_{m}(r) e^{i m \theta},
\end{gathered}
$$

where $J_{m}$ is the Bessel function of the first kind. In the above, we have used the pseudospectral expansion in the azimuthal modes to numerically estimate the surface charge using modes $m$ in the finite range $-K \leq m \leq K$. $K$ is selected to be sufficiently large that spectral contribution of modes with $m>K$ is negligible.

The BC for the electric potential on the surface of the film is $\psi_{2}(r, \theta)=\psi_{3}(r, \theta, z=0)$, and

$$
\begin{aligned}
\psi_{2}(r, \theta) & =\sum_{m=-\infty}^{\infty} \int_{0}^{\infty} J_{m}(k r) A_{m}(k) e^{i m \theta} d k=\sum_{m=-\infty}^{\infty} \hat{\psi}_{2 m}(r) e^{i m \theta} \\
& \approx \sum_{m=-K}^{K} \hat{\psi}_{2 m}(r) e^{i m \theta}
\end{aligned}
$$

The coefficients $A_{m}(k)$ can be calculated with the aid of the Hankel transform,

$$
\int_{0}^{\infty} x J_{m}(k x) J_{m}\left(k^{\prime} x\right) d x=\frac{1}{k} \delta\left(k^{\prime}-k\right) .
$$

Using Eq. (A4), one can solve for the coefficient

$$
A_{m}(k)=k \int_{0}^{\infty} \rho \hat{\psi}_{2 m}(\rho) J_{m}(k \rho) d \rho .
$$

Using the above formulation, the nonlocal relationship between the Fourier coefficients of the surface charge $q$ and the $2 \mathrm{D}$ electric potential $\psi_{2}$ is given by the following integral equation:

$$
\hat{\psi}_{m}(r)=2 \int_{0}^{\infty} k^{2} J_{m}(k r) \int_{0}^{\infty} \rho \hat{\psi}_{2 m}(\rho) J_{m}(k \rho) d \rho d k .
$$

This expression is simplified by the fact that $\hat{\psi}_{2 m}(\rho)=0$ for $\rho<r_{i}$ and $\rho>r_{o}$, so the range of the $\rho$ integration can be restricted to $r_{i} \leq \rho \leq r_{o}$.

Looking back to Eq. (A1), we see that the wave number $k$ describes how rapidly the integrand of $\psi_{3}$ exponentially decreases in the $z$ direction. We can therefore approximate the $k$ integration in Eq. (A6) with a large but finite upper limit $k_{\max }$. To carry out the $k$ integral numerically, we approximate it as a Riemann sum using a small step $\delta k$, with $k_{n}=n \delta k$ and $0 \leq n \leq N_{k}=k_{\max } / \delta k$.

In the pseudospectral method, we only need to evaluate Eq. (A6) at the $N_{c}+1$ Chebyshev collocation points in the radial direction. As described previously, $N_{c}+1$ radial positions $r$ are mapped onto $x$ positions using Eq. (27), with $x_{j}$ $=\cos \left(\pi j / N_{c}\right)$, where $j=0,1,2, \ldots, N_{c}$. The $\rho$ integration in Eq. (A6) can be similarly approximated by a sum so that

$$
\hat{q}_{m}\left(x_{i}\right) \approx 2 \sum_{n=0}^{N_{k}} k_{n}^{2} J_{m}\left(k_{n} x_{i}\right) \sum_{j=0}^{N_{c}} r_{j} \hat{\psi}_{2 m}\left(x_{j}\right) J_{m}\left(k_{n} x_{j}\right) \delta r_{j} \delta k
$$

where $\delta r_{j}$ is an appropriately chosen variable interval. Carrying out the sum over $n$, this can be expressed as a nonlocal, linear transformation between the Fourier-Chebyshev coefficients of $\hat{q}_{m}$ and $\hat{\psi}_{2 m}$,

$$
\hat{q}_{m}\left(x_{i}\right)=\sum_{j=0}^{N_{c}} T_{i j}^{m} \hat{\psi}_{2 m}\left(x_{j}\right) .
$$

We used a $k$ step size of $\delta k=10^{-3}$. It remains to select an appropriate cutoff $k_{\max }$. The best value of $k_{\max }$ was found to be related to the choice of maximum Fourier mode $m=K$. We found that $k_{\max } \sim(1.5-2) \times K$ was sufficient. Varying $k_{\max }$ introduces only very small errors. The integrated charge transport $\mathrm{Nu}$ differs by $\leq 0.3 \%$ at $\mathcal{R}=160$ for simulations with $k_{\max }=40,50,58$, and 70 . We typically used $k_{\max }=50$ to calculate the matrices $T_{i j}^{m}$ for each Fourier mode $m$. The matrix elements depend only on $\alpha, N_{c}$, and $k_{\max }$, and were calculated once at the beginning of the time stepping loop. Equation (A8) was used thereafter to convert $\hat{\psi}_{2 m}$ to $\hat{q}_{m}$. 
[1] E. Bodenschatz, W. Pesch, and G. Ahlers, Annu. Rev. Fluid Mech. 32, 709 (2000).

[2] M. C. Cross and P. C. Hohenberg, Rev. Mod. Phys. 65, 851 (1993).

[3] L. P. Kadanoff, Phys. Today 54 (8), 34 (2001).

[4] S. S. Mao, J. R. de Bruyn, and S. W. Morris, Physica A 239, 189 (1997).

[5] Z. A. Daya, V. B. Deyirmenjian, S. W. Morris, and J. R. de Bruyn, Phys. Rev. Lett. 80, 964 (1998).

[6] Z. A. Daya, V. B. Deyirmenjian, and S. W. Morris, Phys. Rev. E 64, 036212 (2001).

[7] Z. A. Daya, V. B. Deyirmenjian, and S. W. Morris, Phys. Rev. E 66, 015201(R) (2002).

[8] V. B. Deyirmenjian, Z. A. Daya, and S. W. Morris, Phys. Rev. E 72, 036211 (2005).

[9] P. Tsai, Z. A. Daya, and S. W. Morris, Phys. Rev. Lett. 92, 084503 (2004).

[10] P. Tsai, Z. A. Daya, and S. W. Morris, Phys. Rev. E 72, 046311 (2005).

[11] Z. A. Daya, S. W. Morris, and J. R. de Bruyn, Phys. Rev. E 55, 2682 (1997).

[12] V. B. Deyirmenjian, Z. A. Daya, and S. W. Morris, Phys. Rev.
E 56, 1706 (1997).

[13] Z. A. Daya, V. B. Deyirmenjian, and S. W. Morris, Phys. Fluids 11, 3613 (1999).

[14] A. Buka and L. Kramer, Pattern Formation in Liquid Crystals (Springer, New York, 1996).

[15] A. Alonso, M. Net, and E. Knobloch, Phys. Fluids 7, 935 (1995).

[16] A. J. Leadbetter, J. C. Frost, J. P. Gaughan, G. W. Gray, and A. Mosly, J. Phys. (Paris) 40, 375 (1979).

[17] W. F. Langford and D. D. Rusu, Physica A 261, 188 (1998).

[18] U. M. Ascher, S. J. Ruuth, and B. T. R. Wetton, SIAM J. Numer. Anal. 32, 797 (1995).

[19] L. S. Tuckerman and D. Barkley, in Numerical Methods for Bifurcation Problems and Large-scale Dynamical Systems, edited by E. Doedel and L. S. Tuckerman (Springer, Berlin, 2000), p. 435.

[20] R. Peyret, Spectral Methods for Incompressible Viscous Flow (Springer, New York, 2002).

[21] L. N. Trefethen, Spectral Methods in Matlab (SIAM, Philadelphia, 2000)

[22] J. R. Tredicce, G. L. Lippi, P. Mandel, B. Charasse, A. Chevalier, and B. Picque, Am. J. Phys. 72, 799 (2004). 